\title{
Un gesto, una historia. Proyecto artístico como herramienta de identidad y reminiscencia en personas mayores con demencia temprana
}

\section{A gesture a story: artistic project as an identity tool and reminiscence in elderly people with early dementia}

TIPO DE TRABAJO: Comunicación.

PALABRAS CLAVE

Arte, imagen, reminiscencia, demencia temprana.

KEY WORDS

Art, image, reminiscence, early insanity.

RESUMEN

El presente artículo expone un proyecto artístico titulado "Usuario en Línea", compuesto por un archivo configurado por una serie de fotografías de personas en actitud de trabajar en diferentes profesiones, sujetos anónimos pero reconocibles a través de objetos que se establecen en la imagen y dan paso a descubrir cuál es el trabajo que desarrolla la persona representada. Este proyecto posteriormente fue vinculado y dio paso a la elaboración de uno de los talleres realizados en el Centro de Referencia Estatal de Atención a Personas con Enfermedad de Alzheimer y otras Demencias (CREA) del Imserso en Salamanca. El Taller denominado "Un gesto una Historia" permitió la conexión con la historia de vida de los participantes y en concreto de sus actividades laborales, las cuales fueron descritas e incluso representadas gráficamente, con el fin de evocar recuerdos y vivencias pasadas. Las obras seleccionadas del proyecto, fueron analizadas con el objetivo de mantener activo el cerebro del usuario, es decir, invitan al espectador a un juego de percepción visual, (Arnheim, 2005), en el que se utiliza el Arte de Camuflaje (Méndez Baiges, 2007) para crear la imagen, donde las personas mayores con demencia pueden ver objetos que en su conjunto forman una imagen de una persona desarrollando una actividad de la vida diaria (AVD) como es el trabajar. Estas obras nos permiten abrir el diálogo hacia varios caminos, permitiéndonos averiguar cuál y cuantos objetos se esconden tras el cuadro mediante el análisis de su silueta. El ejercicio precisa de atención sostenida y centrada en cada objeto que compone la figura. Este juego visual (Winnicott, 2008) ha sido utilizado con participantes en Fase leve y leve-moderada, obteniendo resultados positivos por el interés que despierta el juego visual y adivinanza entre los y las participantes, generándose episodios de humor y risas compartidas.

\section{ABSTRACT}

This article presents an artistic project "Online User", consisting of a file configured by a series of photographs of people in an attitude of working in different professions, subjects anonymous but recognizable through objects that make up the image and give way to discover what is the work that the represented person develops. This project was subsequently linked and led to the development of one of the workshops held in Centro de Referencia Estatal de Atención a Personas con Enfermedad de Alzheimer y otras Demencias (CREA) del Imserso en Salamanca. The workshop called "A gesture a History" allowed the connection with the life history of the participants and in particular of their work activities, which were described and even represented graphically, in order to evoke memories and past experiences. The selected works of the project, were analyzed with the objective of keeping the user's brain active, that is, they invite the viewer to a game of visual perception, (Arnheim, 2005), in which the Art of Camouflage is used (Méndez Baiges, 2007) to create the image, where elderly people with Dementia can see objects that together form an image of a person developing an activity of daily life (ADL) such as working. These works allow us to open the dialogue towards several paths, allowing us to find out which and how many objects are hidden behind the painting by analyzing their silhouette. The exercise requires attention and focused 
on each object that makes up the figure. This visual game (Winnicott, 2008) has been used with participants in the mild and mildmoderate phase, obtaining positive results due to the interest that the visual game and riddle arouse among the participants, generating episodes of humor.

\section{INTRODUCCIÓN}

La intervención y mediación artística para contribuir a la mejora de pacientes con demencia, es una realidad que ya cuenta con una serie de evidencias cientificas (Miller \& Hou 2004; Gray, 2011; Stuckey \& Nobel, 2010). Como sostienen, Chancellora, Duncanc \& Chatterjeea (2014:4), esas evidencias se resumen en: la intervención con Arte se basa en las capacidades preservadas en lugar de en la discapacidad que padece la persona. A nivel internacional el primer referente en proyectos de estas características, es el Proyecto, Meet me at MoMA. Proyecto implementado por el Museo de Arte Moderno de Nueva York, más conocido como MoMA.

A nivel nacional como principal exponente, nos encontramoscon el Proyecto Muban Alzheimer,cuyo equipo formado por Delgado, Hervas y Arnardóttir (2014) han llevado a cabo una serie de experiencias de talleres artístico-museísticas, mediante determinadas herramientas de investigación, así como un programa informático desarrollado por Paul Ekman (2003) denominado Micro Expression Training Tool (METT), para el reconocimiento de microexpresiones faciales de las emociones de los usuarios, que sirven al investigador-observador analizar y sistematizar datos.

Actualmente, las intervenciones relacionadas con el Arte y enfocadas a la mejora de la calidad de vida de usuarios con Demencia temprana se han desarrollado exponencialmente en los últimos años. Un ejemplo más de este tipo de proyectos es el proyecto "Usuario en Línea" (López-Méndez, 2016), compuesto por un archivo de carácter artografico, (Iwin y De Cosson, 2014), configurado por una serie de fotografías de personas en actitud de trabajar en diferentes profesiones; sujetos anónimos pero reconocibles a través de objetos que configuran la imagen camuflada y dan paso a descubrir cuál es el trabajo que desarrolla la persona representada. (Véase, Figura.1).

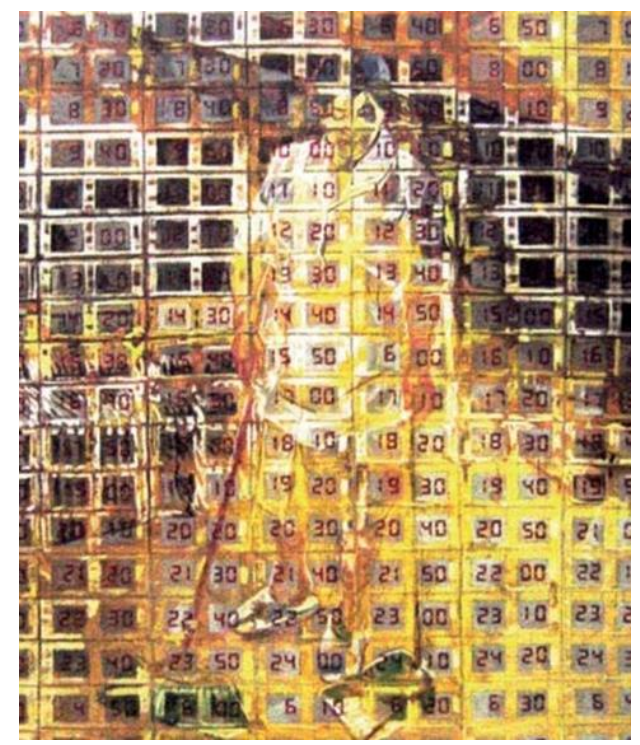

Figura.1. Camarera de Piso. 2007.Óleo sobre lienzo 150 x 150 cm. Fuente: Lorena López.

El archivo nos permitió implementar el taller "Un Gesto una Historia”, un proyecto artístico que pone en valor una actividad educativa, lúdica y creativa empleando como temática el concepto trabajo, como actividad a la que nos dedicamos una importante parte de nuestra vida y que es huella y memoria humana en el trascurso del tiempo. El taller se llevó a cabo con personas que padecen Demencia temprana en fase leve, en el Centro de Referencia Estatal de Atención a Personas con Enfermedad de Alzheimer y otras Demencias (CREA) del Imserso en Salamanca.

Durante el proceso, nos centramos en las profesiones de los y las participantes, lo que nos permitió establecer vínculos y relaciones entre ellos y nosotros, entre la sociedad del s.XX y la que nos corresponde s.XXI. Partiendo de las ideas recogidas, pudimos plantearles el cambio de paradigma en relación al trabajo, estableciendo diferencias entre el Artista artesano y el Artista contemporáneo. La herramienta, para elaborar el discurso de los participantes, fue el empleo de la Pintura, una técnica que determinados autores apuntan que ha llegado su "fin", un supuesto "fin" que también ahonda Rifkin en su obra de 1996, "El fin del trabajo". 
Estableciendo una paralelismo y revisión de ambos conceptos (Pintura-Trabajo) a lo largo de la Historia del Arte, a través de una serie de obras pictóricas de artístas legitimados y de la autora de este artículo (Veáse, tabla 1). Se pretendió que el espectador participase como creador y adquieriera conocimientos artísticos que le permitiesen explorar en sus biografías y experimentar emociones y sensaciones.

\begin{tabular}{|c|c|}
\hline ARTÍSTAS & OBRA/TÍTULO \\
\hline Diego Velázquez & Las Meninas (1656) Oleo sobre lienzo. 3,18 x 2,76 cm. Museo del Prado.Madrid. \\
\hline Millet. & El Angelus -Oración (1859). Óleo sobre lienzo. M66 x 5,5cm. Museo de Orsay.París. \\
\hline Van Gogh. & Campesino con mujer plantando pata- (1884) Óleo sobre lienzo.. 70 x170 cm.Amsterdam. \\
\hline Piotr Strojeu. & Complejo Textil de Kirovabad. (1937).Óleo sobre lienzo. Moscú. \\
\hline Diego Rivera. & Vendedora de Flores. (1941.) Óleo sobre tela. Mexico. \\
\hline ARTÍSTA/EDUCADOR/INVESTIGADOR & OBRA/DISPOSITIVO/TITULO \\
\hline Lorena López-Méndez & $\begin{array}{l}\text { Camarera de Piso (2008). Óleo sobre lienzo, } 150 \text { x } 150 \mathrm{~cm} \text {. } \\
\text { Minero (2009). Óleo sobre lienzo, } 100 \text { × } 100 \mathrm{~cm} \text {. } \\
\text { Agricultor (2010). Óleo sobre lienzo, } 150 \text { × } 150 \mathrm{~cm} \text {. }\end{array}$ \\
\hline
\end{tabular}

Tabla 1. Relación de obras.

\section{METOdOLOGÍA}

El programa artístico se configuró en siete sesiones de una hora y treinta minutos en formato grupal ( 2 grupos de 8 usuarios máximo). La intervención se realizó entre las 15:00 y 16:45 el primer grupo y entre las 17:00 y las 18:45 p.m el segundo. El primer grupo en un espacio designado específicamente para la actividad y aislado de las unidades de convivencia y el centro de día.

Las sesiones se llevaron a cabo con la presencia de una investigadora especializada en mediación artística, para este perfil de participantes, encargada de diseñar, implementar y coordinar el taller, dos educadores sociales y una auxiliar de clínica de apoyo en la fase procesual del mismo.

Los objetivos terapéuticos implementados durante las sesiones fueron pautados en colaboración con profesionales del centro CRE Alzhéimer. (Veáse, figura 2).

\section{Objetivos artísticos}

- Reflexionar sobre el trabajo como actividad.

- Dialogar sobre el concepto de artista hoy en día.

- Conocer una de las técnicas artísticas más utilizadas a lo largo de la historia, como es la pintura.

- Recordar los oficios que tenían los participantes a través de la Reminiscencia.

\section{Objetivo terapéuticos}

- Mejora de la motivación y atención a través del diálogo.

- Activación de recuerdos y emociones positivas a través del visionado de obras de Arte.

- Fomento de la resiliencia por medio del sentimiento de capacidad de crear una obra artística propia.

- Mejora de la calidad de vida.

- Estimular habilidades motrices y funciones cognitivas. 


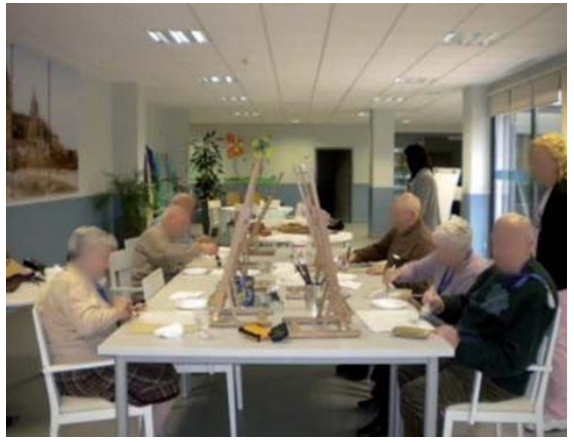

Figura 2. ESPACIO DE TRABAJO. Fase Imprimación de tablas. Fuente: Lorena López.

La metodología que se implementó de carácter cualitativo, se basó principalmente en una observación participante, centrada en entrevistas no estructuradas, así como en el desarrollo de un registro a modo de notas de campo y un archivo fotográfico durante las sesiones, que posteriormente fueron analizados por medio del uso del programa de análisis de datos atlas.ti (versión 7).

\section{DESARROLLO}

El desarrollo del taller se llevó a cabo en siete sesiones como apuntamos anteriormente, en las que se implementaron tres fases que se repitieron en cada una de las sesiones. Las fases consisten en, una fase de apreciación y visualización de obras de arte relacionadas con el concepto trabajo o labores, una fase de producción en la que los participantes desarrollaban su propia obra y por último, una fase de debate, en la que los usuarios debatían sobre sus resultados.

La primera sesión, comenzó con la fase de apreciación y el visionado de manera virtual del cuadro de "Las Meninas" de Diego Velázquez, (1656), para iniciarnos en un breve recorrido por la Historia de la Pintura, pues la mayoría de los participantes no tenían conocimientos respecto a la del Arte Actual.

Seguidamente se procedió a la realización de un debate por parte de los participantes en relación a la serie de imágenes de Pinturas que habían visualizado, ya que en su mayoría se trataban de representaciones visuales cuya temática era" El trabajo", un tema muy cercano para ellos, más aún si se trata del trabajo y quehaceres del campo como hilo conductor. Enlazándolo con la faena que realizaba el hombre y la mujer en la prehistoria, los cuales representaban en las cuevas y gracias a ellos hoy conocemos su historia.

El debate se generó a partir de una serie de preguntas ¿Qué es para ti la pintura? ¿Nos permite retratar un acontecimiento de un recuerdo? En este punto introducimos la temática bajo la que giraban las obras. Las preguntas que generamos como continuación del debate, fueron las siguientes: ¿Qué tema veis en las obras? ¿Qué hacen? ¿Qué objetos aparecen? y ¿Para qué sirven?

Introducido el tema del "Trabajo" a los participantes, incidiendo en que dialogasen en torno a ese tema, se les preguntó: ¿En que trabajaban ellos? ¿Cómo desempeñaban su trabajo? ¿Dónde? -De tal manera que rescatemos sus recuerdos y anécdotas vividas en su jornada laboral, a la vez que también les exponíamos nuestra visión del trabajo, siempre desde un punto de vista positivo, generando conocimientos, ya que muchos de los trabajos que ellos han desempeñado, se encuentran muy alejados de nosotros al vivir en un mundo donde las máquinas comen terreno al trabajador.

Algunos participantes mostraron entusiasmo volviendo a recordar acciones físicas que estaban vinculadas a su trabajo. El cuerpo parece tener también memoria y la participante $\mathrm{T}$, empezó mover sus manos como si estuviera tecleando en su máquina de escribir o el participante M. nos narró el esfuerzo físico que tenía que hacer para arar sus tierras y como se debía de coger el azadón. Este ejercicio constituyó una excelente forma de recordar las capacidades que tuvieron en el pasado.

Finalmente, en la fase producción, los participantes realizaron su propia obra pictórica, en la que tenían. como punto de partida la temática del trabajo para su realización. Una vez concluidas todas las obras de los participantes, fueron expuestas en una presentación digital, de tal manera que pudieron verlas y comentarlas entre los propios participantes y el personal del Centro.

La propuesta se planteó de la siguiente manera ¿Cuál era tu trabajo, y cómo podríamos representarlo? Para la realización de la pintura, se optó por utilizar como soporte tablillas de madera, que previamente se imprimaban con una base de jesso que podía ser de 
color si lo mezclaban con alguno de los colores que les ofreciamos. Esto nos permitió dar a conocer a los participantes la profesión del Pintor y cómo también esta ha cambiado.

Aplicada la imprimación y una vez seca, podían proceder a pintar lo que ellos recordaban sobre su trabajo. La mayoría de los participantes escogieron realizar campos de color. Por lo tanto, se centraron en la abstracción como forma de expresión. (Véase, figura 3).

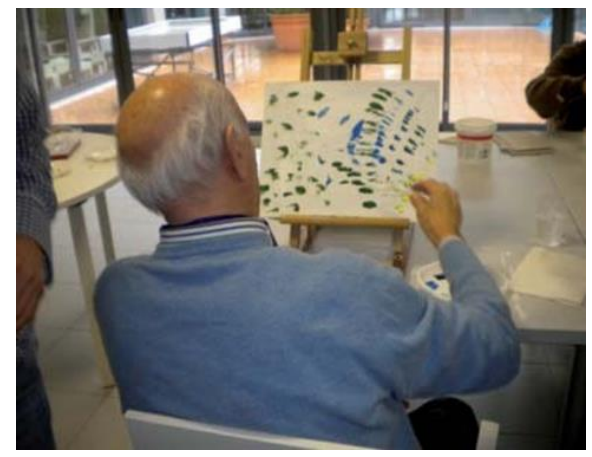

Figura 3. Encajes. Marzo 2011. Fuente: Lorena López.

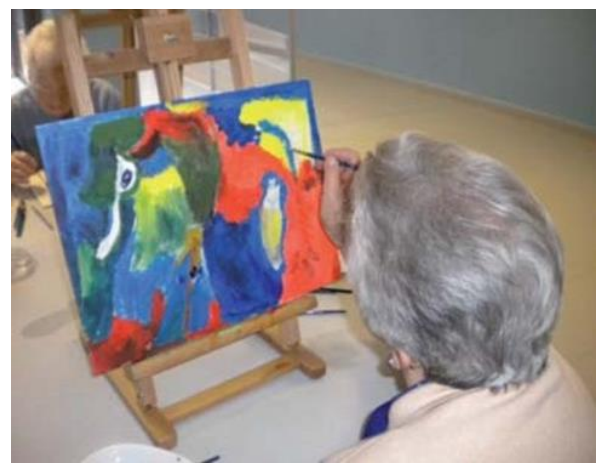

Figura 4. Últimas pinceladas. Fuente: Lorena López.

Las obras resultantes de los participantes sin lugar a dudas tienen que ver con el concepto abstracción o puntillismo. Corrientes artísticas que tratamos a lo largo de las sesiones. Asimismo, cabe destacar que en ocasiones los participantes se "copiaban" unos a otros en cuanto a estilos. Al ver nuestra aprobación y considerando la forma de trabajar del compañero como valida, el resto de participantes continuaron realizando el mismo proceso.

Al final se estableció un debate en relación a los resultados obtenidos y las dificultades planteadas en el trascurso de la realización.

\section{CONCLUSIONES}

En un principio, varios usuarios tenían la impresión de que no iban a ser capaces de representar un concepto en sus tablillas y se resistían a participar. Finalmente, gracias al entusiasmo que mostraron el resto de usuarios, los que al inicio se manifestaban reticentes se incorporaron. Lo más importante fue el ambiente creador, agradable y relajado en el que convertimos el taller, en el que los recuerdos tuvieron más relevancia que el mérito artístico de lo que se estaban realizando.

La actividad se utilizó como una forma de liberación, para algunos participantes. El hecho de pintar fue una forma de explicar una idea cuando las palabras no les venían a la mente con fluidez. La parte procesual del taller, al ser realizada mediante el uso de la técnica reina de los procedimientos artísticos, la pintura, obtuvo una gran acogida por parte de los participantes, incluso uno de ellos, concretamente J.M, había sido pintor, hecho que se manifestó durante el taller, ya que no era normal la destreza que poseía al utilizar el pincel como herramienta de trabajo. Otro caso de interés es el de $S$, un agricultor que jamás había cogido un pincel, pero que sin embargo supo muy bien representar su trabajo entorno a campos de color que simulaban la tierra arada. 
Sin embargo, no sólo hablamos de trabajo, sino también de ocio, un ocio que como apunta Greenberg (1979), en su obra "Arte y Cultura" (Ensayos críticos), es tanto una función como un producto del trabajo, y que cambia al cambiar el trabajo. También Artistoteles (Greenberg, 1979:35), afirmaba que el primer principio de toda acción es el ocio. Tanto trabajo como ocio son necesario, pero el ocio es mejor y es su fin...produce placer y felicidad y alegría de vivir. Este es el propósito de nuestros talleres, hacer experimentar al participante un disfrute convertido en ocio.

Como decía el participante T, que se encontraba en fase leve y aún se podía mantener conversaciones distendidas, "el fenómeno de la industrialización nos ha facilitado la vida en algunos temas como en el ocio pero en otros nos dificulta, sobre todo a los mayores para desenvolvernos...". En cuanto al participante A. sostenía que "Respecto al Arte, ahora es nuestro tiempo de ocio con vosotros, nuestro saber popular es importante para poder dejar legado en nuestra sociedad, aunque con nuestra enfermedad poco podemos ir dejando". esta conversación el participante S. apuntó que el "cuando era joven, no tenía apenas ocio, siempre estaba en el campo, labrando, trabajando duro para poder vivir y las circunstancias le impedían disfrutar del Arte como estaba haciendo en el taller".

Finalizadas las sesiones, concretamente un número de siete y efectuado su análisis, pudimos observar que, tanto los objetivos terapéuticos marcados como los artísticos se lograron. Asimismo, cabe destacar como el diseño de un elemento que les llamase la atención como detonante, sirvió de llave maestra para poder captar la atención de los participantes. Consideramos de suma importancia tratar un tema tan cotidiano como es el "trabajo" para poder enlazar el Arte Contemporáneo con sus vidas y de esta manera traer a sus mentes su vida pasada. De esta forma aplicamos la teoría de la reminiscencia, que precisamente consiste en compartir recuerdos de la experiencia vital de los participantes para poder debatir sobre el pasado.

Explicar los rudimentos de la Pintura, como trabajo y práctica artística fue un éxito, pues en las sesiones siguientes, eran capaces de desenvolverse con total independencia y autonomía, tanto en el uso de las herramientas como en el tema conceptual a tratar.

La inclusión del concepto trabajo cotidiano y proceso pictórico a la par, supuso una revelación, pues muchos de ellos parecía que hubiesen pintado toda su vida, es más, en el análisis del resultado de sus obras, se pueden extraer y destacar parecidos con los resultados obtenidos por artistas Expresionistas abstractos y de las primeras vanguardias.

Los y las participantes trabajaron de manera individual acompañados de un educador que les iba aportando instrucciones en el caso de que las requirieran, aunque en su mayoría a lo largo del transcurso de las sesiones ya no precisaban de la ayuda del educador; salvo para solicitar algún color en especial que se les hubiese agotado en la paleta.

Los participantes mostraron las mismas dificultades que podría haber mostrado cualquier adulto que no esté al tanto del uso de técnicas artísticas. (Ullán et al, 2012). Por tanto, pudimos comprobar que las sesiones que se sirven del uso de procesos artísticos tradicionales, más sencillos de entender para los participantes, son recomendables para trabajar con participantes con Alzhéimer y otras Demencias.

Con actividades como la descrita anteriormente consideramos que es posible mejorar la calidad de vida de los pacientes con demencia temprana, porque estimulamos la comunicación cotidiana entre los participantes, ayudamos a crear sensación de bienestar. A su vez activamos emociones positivas, generamos nuevas relaciones afectivas, fomentamos la autoestima, preservamos capacidades cognitivas, permitiéndonos, ejercitar psicomotricidad fina y gruesa, etc.

Por lo tanto, consideramos que los usuarios del Centro tienen derecho a participar de la vida cultural y artística y para ello diseñamos talleres de creación en los que puedan reflexionar sobre el mundo en el que vivimos, conectando su realidad con lo que sucede dentro y fuera del Centro.

El taller "Un Gesto una Historia" fue una experiencia enriquecedora, pues es importante trabajar mediante proyectos, en los que el participante genere su propio discurso en base a una temática, que le sea cercana a su vida cotidiana, de tal manera que, al fomentar la repetición, logramos que el recuerdo perdure, ya que cuando aprendemos, la práctica es lo que culmina la asimilación de conocimientos. (López-Méndez,2014, 2017).

Para finalizar apuntamos una de las frases mencionadas por una de los participantes: "Yo jamás había pintado, pero parece que lo he hecho toda la vida" Participante S. 
López Méndez, Lorena

Un gesto, una historia. Proyecto artístico como herramienta de identidad y reminiscencia en personas mayores con demencia temprana IV Congreso INTERNACIONAL DE INVESTIGACIÓN EN ARTES VISUALES ANIAV 2019

IMAGEN [N] VISIBLE]

http://dx.doi.org/10.4995/ANIAV.2019.8963

\section{FUENTES REFERENCIALES}

Arheim, R. (1985). El Pensamiento Visual. Buenos Aires: Eudeba.

Chancellor, B., Duncan, A y Chatterjee, A. (2014). Review Art Therapy for Alzheimer's Disease and Other Dementias. Journal of Alzheimer's Disease, 39 (2014), 1-11. https://doi.org/10.3233/JAD-131295

Delgado, M., Hervás, R.M. y Arnardóttir, H. (2014). Arte para evocar emociones y recuerdos en personas con Alzheimer. En Chafer Bixquert, T. (2014), I Congreso Internacional de Espacios de Arte y Salud. Hospital de Dénia 2012. Valencia: Editorial Universitat Politècnica de València.

Gray, A.E. (2011). Expressive arts therapies:Working with survivors of torture. Torture 21, 39-47.

Greenberg, C. (1979). Arte y Cultura. Ensayos Críticos. Barcelona: Gustavo Gili.

Irwin, R y De Cosson, A. (2004). A/r/tography: Rendering Self Through Arts-based Living Inquiry. Canada: Pacific Educational Press.

López-Méndez, L. (2014). Educación artística en personas con Demencia Temprana. International Journal of Developmental and Educational Psychology. INFAD Revista de Psicología, 1(1), 527-534.

López-Méndez, L. (2016). Usuario en línea: Nuevas formas de representar el trabajo en el Arte. Madrid: Editorial Académica Española.

López-Méndez, L. (2017) Programa retales de una vida del Proyecto AR.S Alzheimer: Herramientas para dialogar y estimular recuerdos a través del Arte. Arte, Individuo y Sociedad, 29(Núm. Especial), 139-158. http://dx.doi.org/10.5209/ARIS.53338

Méndez Baiges, M. (2007). Camuflaje: engaño y ocultación en el arte contemporáneo. Madrid: Siruela.

Miller, B.L., y Hou, C.E. (2004). Portraits of artists: Emergence of visual creativity in dementia. Arch Neurol 61, 842-844.

Stuckey, H.L., y Nobel, J. (2010). The connection between art, healing, and public health: A review of current literature. Am J Public Health, 100, 254-263.

Rifkin, J. (2010). El fin del trabajo: nuevas tecnologías contra puestos de trabajo. El nacimiento de una nueva era. Madrid: Editorial Planeta.

Ullán, A.M., Belver, M.H., Badia, M., Moreno, C., Garrido, E., Gómez-Isla, J., Gonzaléz-Ingelmo, E., Delgado, J., Serrano, I., Herrero, C., Manzanera, P., y Tejedor, L. (2012). Contributions of an artistic educational program for older people with early dementia: An exploratory qualitative study. Dementia, 12(4), 425-446. https://doi.org/10.1177/1471301211430650.1-22

Winnicott, D. W. (1999). Realidad y juego. Barcelona: Gedisa. 